\title{
Caseous Calcification of the Mitral Valve Annulus Simulating an Intramyocardial Mass at Autopsy
}

\section{Rizkalla $\mathbf{H}^{1 *}$, O'Briain $\mathbf{S}^{1}$ and Sheppard $\mathbf{M}^{2}$}

${ }^{1}$ Department of Pathology, St. James's Hospital, Dublin 8, Ireland

${ }^{2}$ Department of Cardiology, Royal Brompton Hospital, London, UK

*Corresponding author: Hala Rizkalla, Department of Pathology, St. James's Hospital, James's Street, Dublin 8, Ireland, Tel: 353-01-410-3000; E-mail: halafathy25@hotmail.com, hala.fathy553@gmail.com

Received: January 01, 2016; Accepted: April 29, 2016; Published: May 09, 2016

Copyright: ( 2016 Rizkalla $\mathrm{H}$, et al. This is an open-access article distributed under the terms of the Creative Commons Attribution License, which permits unrestricted use, distribution, and reproduction in any medium, provided the original author and source are credited.

\begin{abstract}
Mitral annular calcification is an asymptomatic chronic degenerative process of the mitral valve, which can occur in the elderly population, associated with atherosclerosis. When it is extensive it is termed caseous calcification of the mitral valve annulus. We illustrate the pathology of this entity in the heart in an 80 -year-old man, in whom it presented as a large intramyocardial cystic mass found at autopsy.
\end{abstract}

\section{Introduction}

Mitral annular calcification is an asymptomatic chronic degenerative process of the mitral valve, which can occur in the elderly population, associated with atherosclerosis [1]. It may also occur in patients with end stage renal failure or other metabolic disorders that result in abnormal calcium deposition. The entity described is a rare finding termed liquifactive necrosis or caseous calcification of the mitral valve annulus (CCMA). The prevalence of CCMA is reported to be $0.6 \%$ of mitral annular calcification and $0.06-0.07 \%$ of the population referred for echocardiography $[2,3]$. In autopsy studies, its prevalence rises to $2.7 \%$ [1].

\section{Case Description}

An 80-year-old man with no significant past medical history, presented to the emergency department, after being found collapsed at his home. He was pronounced dead on arrival. At autopsy, the body mass index was 17.6 and the heart weight was $430 \mathrm{~g}$. There was triple coronary vessel atherosclerosis with over $90 \%$ stenosis and evidence of old myocardial infarction.

In addition there was a palpable mass in the posterobasal aspect of the left ventricle. This $6 \mathrm{~cm}$ spherical mass was evident on opening the left ventricle and associated with thickening of the overlying endocardium and fibrous adhesions of the pericardium externally. The mass was located in the myocardium beneath the posterior mitral valve annulus, between the coronary sinus and the posterior mitral valve leaflet it did not involve the leaflet but extended downwards to involve the posterior left ventricular myocardium. The cyst contents consisted of necrotic material that had a pastry cream-gray appearance (Figure 1). There was no plane of dissection outside the cyst [1].

Histological examination of the left ventricular myocardial wall revealed a fibrous-walled cyst with extensive calcification. Mixed inflammatory cells were seen adjacent to the advancing edge of the cyst wall including mast cells and multinucleated giant cells almost granulomatous in appearances in some fields. No cyst lining was identified. Amorphous granular debris was seen as cyst content but no cellular component was present (Figures 2 and 3 ).

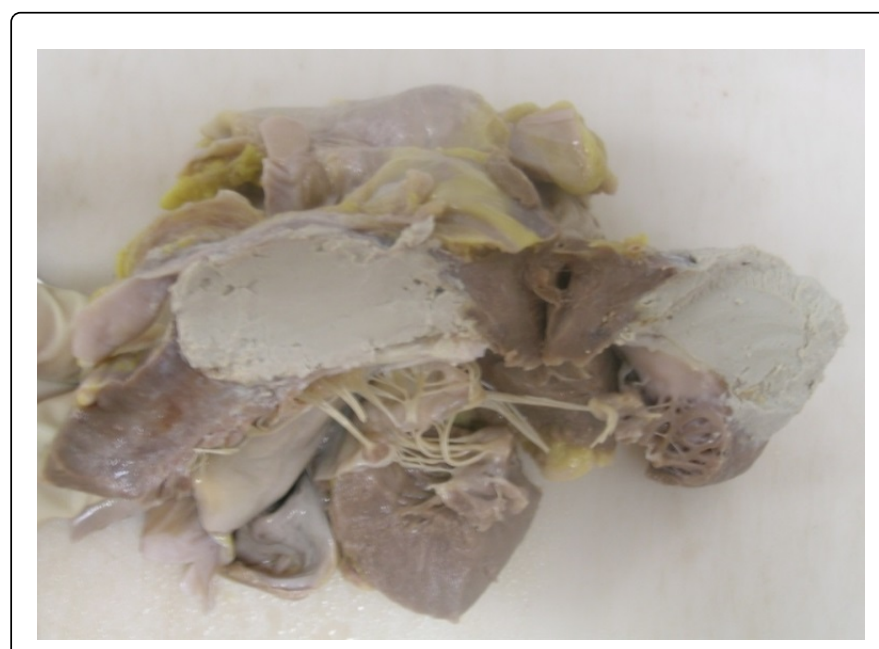

Figure 1: Narcotic material on the cyst.

Histological examination of the left ventricular myocardial wall revealed a fibrous-walled cyst with extensive calcification. Mixed inflammatory cells were seen adjacent to the advancing edge of the cyst wall including mast cells and multinucleated giant cells almost granulomatous in appearances in some fields. No cyst lining was identified. Amorphous granular debris was seen as cyst content but no cellular component was present (Figures 2 and 3). Special stains for microorganisms and for acid fast bacilli were negative. Of note was a small, $0.5 \mathrm{~cm}$ similar calcified focus in the upper lobe of left lung, indicating that this person was a 'calcifier' [2].

The intramyocardial mass was consistent with mitral annular calcification with progression to caseous necrosis forming a mass mimicking a tumour or a tuberculoma within the myocardium. Caseous calcification represents a rare evolution of a calcified mitral ring, due to liquifactive transformation of the inner material which can lead to an erroneous diagnosis of cardiac tumour or abscess. 
Citation: Rizkalla H, O'Briain S, Sheppard M (2016) Caseous Calcification of the Mitral Valve Annulus Simulating an Intramyocardial Mass at Autopsy. J Cytol Histol 7: 1000406. doi:10.4172/2157-7099.1000406

Page 2 of 2

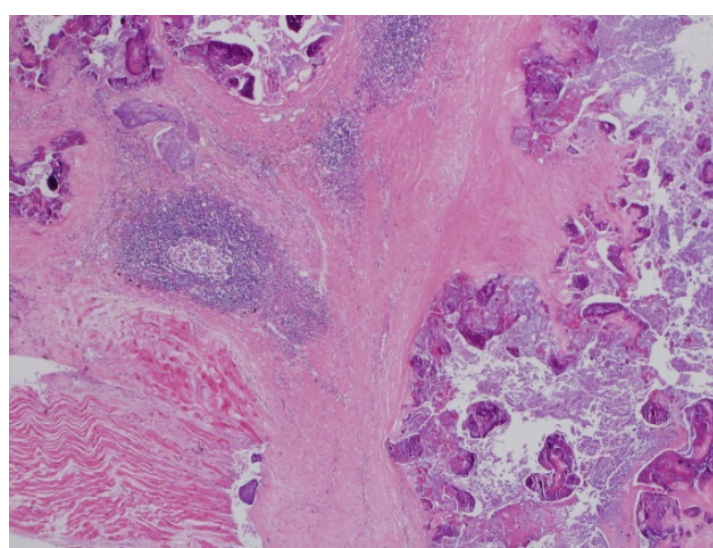

Figure 2: Histological examination of the left ventricular myocardial wall.

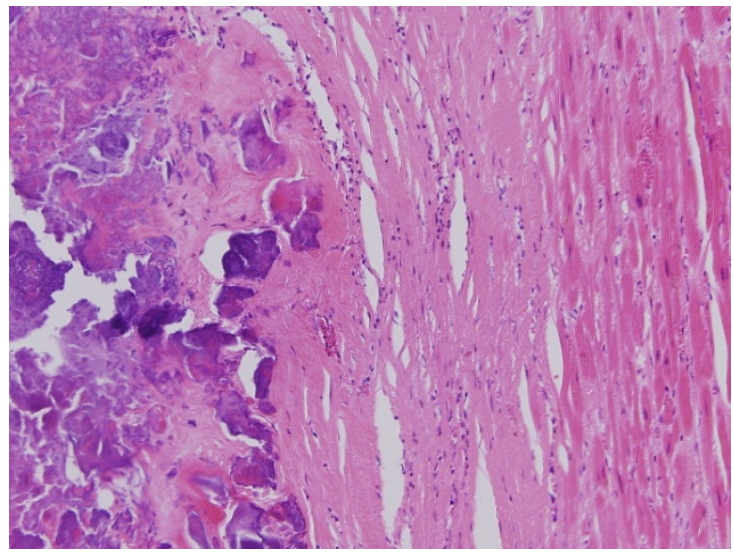

Figure 3: Debris on the cyst.
The intramyocardial mass was consistent with mitral annular calcification with progression to caseous necrosis forming a mass mimicking a tumour or a tuberculoma within the myocardium. Caseous calcification represents a rare evolution of a calcified mitral ring, due to liquifactive transformation of the inner material which can lead to an erroneous diagnosis of cardiac tumour or abscess. The typical location of a calcified cyst with well-defined borders, characteristic internal contents and involvement of the mitral annulus is characteristic of CCMA. The distinction between CCMA and mitral annulus abscess should be based on the clinical presentation, the lack of calcification and histological and or microbiological findings. Calcified hydatid cysts in the heart usually shell out from the surrounding myocardium relatively easy and they show characteristic histological findings. Further differential diagnosis of lesions in this location would include lipomatosis of the atrioventricular groove and benign pericardial cysts [3].

CCMA is well described in cardiothoracic and echocardiographic studies but appears to be underappreciated in pathology reviews. We illustrate the pathology of the heart in an 80-year-old man, in whom a large intramyocardial cyst filled with large amount of pasty or "toothpaste-like" material was found at autopsy. General pathologists should be familiar with this rare form of periannular calcification, to avoid misinterpretation of CCMA as a degenerate cardiac neoplasm, an abscess or a tuberculoma.

\section{References}

1. Pomerance A (1970) Pathological and clinical study of calcification of the mitral valve ring. J Clin Pathol 23: 354-361.

2. Harpaz D, Auerbach I, Vered Z, Motro M, Tobar A, et al. (2001) Caseous calcification of the mitral annulus: a neglected, unrecognized diagnosis. J Am Soc Echocardiogr 14: 825-831.

3. Deluca G, Correale M, Leva R, Del Salvatore B, Gramenzi S, et al. (2008) The incidence and clinical course of caseous calcification of the mitral annulus: a prospective echocardiographic study. J am Soc Echocardiogr 7: $828-833$. 